\title{
Prevalence of and Risk Factors for Cerebral Microbleeds in Moyamoya Disease and Syndrome in the American Population
}

\author{
Nadeem I. Khan ${ }^{a}$ Ali A. Saherwala ${ }^{a}$ Mo Chen $^{a}$ Sepand Salehian ${ }^{c}$ \\ Hisham Salahuddina $^{a}$ Babu G. Welch ${ }^{\text {b, c }}$ Marco C. Pinho ${ }^{c}$ Ty Shang ${ }^{a}$ \\ aDepartment of Neurology, University of Texas Southwestern Medical Center, Dallas, TX, \\ USA; b Department of Neurosurgery, University of Texas Southwestern Medical Center, \\ Dallas, TX, USA; ' Department of Radiology, University of Texas Southwestern Medical \\ Center, Dallas, TX, USA
}

\section{Keywords}

Stroke $\cdot$ Moyamoya syndrome $\cdot$ Cerebral microbleeds $\cdot$ Population studies

\begin{abstract}
Background and Purpose: Cerebral microbleeds (CMB) are reported to be frequent in moyamoya disease (MMD) and moyamoya syndrome (MMS) in the Asian population. It is associated with an increased risk of intracerebral hemorrhage. The significance of CMB in MMD/ MMS in non-Asian populations has not been well established. Our study aimed to investigate the prevalence of $\mathrm{CMB}$ in MMD/MMS in a moymoya cohort with a majority of non-Asians and to identify risk factors for developing a $\mathrm{CMB}$ and its predictive value for subsequent vascular events. Methods: The moyamoya database was compiled by screening for MMD/MMS among patients admitted to the Zale-Lipshy University Hospital at the University of Texas Southwestern Medical Center. We identified and analyzed data of 67 patients with MMD or MMS. Patients were characterized as $\mathrm{CMB}+$ or $\mathrm{CMB}$ - based on $\mathrm{MRI}$ findings. In $\mathrm{CMB}+$ patients, the total number and location of $C M B$ were identified. Univariate and multivariate logistic regression were used to identify risk factors for developing $\mathrm{CMB}$ and whether $\mathrm{CMB}$ are associated with the development of subsequent vascular events. Results: Out of a total of 67 patients, 11 (16\%) had CMB. Males had significantly higher odds of having CMB as compared to females (OR 1.76; 95\% Cl 1.40-24.3, $p=0.021$ ). The incidence of CMB was also associated with age at diagnosis (mean age of $\mathrm{CMB}+$ patients vs. $\mathrm{CMB}$ - patients: 44 vs. 34 years, respectively, $p=$ $0.024)$, smoking $(p=0.006)$, and hemorrhagic stroke at presentation $(p=0.034)$. Logistic re-
\end{abstract}


gression with multivariate analysis found that gender and age at diagnosis remained statistically significant. New ischemic events occurred in 2 (20\%) out of $10 \mathrm{CMB}+$ patients and 13 (23\%) out of $55 \mathrm{CMB}$ - patients, respectively $(p=0.79)$. While $2(3 \%) \mathrm{CMB}-$ patients had a new cerebral hemorrhage during follow-up, none of the $\mathrm{CMB}+$ patients did. Conclusions: $\mathrm{CMB}$ are less prevalent in MMD/MMS in the USA than in Asia. An older age at diagnosis and male gender were associated with $\mathrm{CMB}$. The presence of $\mathrm{CMB}$ was not associated with an increased risk of a subsequent ischemic or hemorrhagic stroke.

(C) 2019 The Author(s)

Published by S. Karger AG, Basel

\section{Introduction}

Moyamoya disease (MMD) is a chronic progressive cerebrovascular disease defined by bilateral or unilateral stenosis of arteries around the circle of Willis with subsequent development of collateral blood vessels. First identified in Japan, MMD is less frequently reported in Western countries, with an estimated incidence of 0.086 per 100,000 [1]. The idiopathic form of MMD has been linked to several genetic loci in the Asian population, including the RNF213 gene in chromosome 17 [2]. Patients with moyamoya syndrome (MMS) have angiographic findings similar to those of MMD patients but with well-known associated medical conditions, such as atherosclerosis [3], diabetes, infection [4], sickle cell disease [5-7], neurofibromatosis type 1 [8], tuberous sclerosis [9], Sturge-Weber syndrome [10], Down syndrome [11], and Turner syndrome [12].

The vascular pathology of MMD is characterized by progressive stenosis and occlusion of the internal carotid artery, the proximal middle cerebral artery, and the anterior cerebral artery, and rarely the posterior cerebral artery, with subsequent development of parenchymal, leptomeningeal, and/or transdural collateral vessels. Microscopically, stenotic or occlusive large vessels show fibrocellular thickening of the intima, replication of the internal elastic lamina, and thinning of the media $[13,14]$. These collateral vessels have thin walls that are prone to hemorrhage, aneurysm, and thrombosis. MMS patients in Asian populations present with intracerebral hemorrhages more commonly than do MMS patients in Western populations, who present more often with TIA and ischemic stroke $[15,16]$.

Cerebral microbleeds (CMB) are small foci of signal abnormality detected as hypointensities in MRI T2*-weighted standard gradient echo (GRE) or susceptibility-weighted images (SWI) with a high sensitivity. CMB have been identified as potential diagnostic and prognostic markers in multiple disease processes, such as stroke, intracranial arteriopathy, brain aging, and brain trauma. CMB are more common in diseased brains than in healthy controls (up to $36 \%$ vs. less than $4.5 \%$ ). The presence of CMB increases the risk of new cerebrovascular events, stroke-related death, and hemorrhagic conversion of an ischemic stroke. In addition, CMB are a predictor of future stroke in patients with transient ischemic attacks [17].

To the best of our knowledge, the significance of CMB in MMD/MMS in non-Asian populations has not been well established. There has only been 1 study of the central European population of MMD/MMS in Germany, in which out of 101 patients $13(12.9 \%)$ had identified CMB [18]. Our study aimed to investigate the prevalence of CMB in MMD/MMS in our referral population of patients in the USA, identify risk factors for developing $\mathrm{CMB}$, and understand the predictive value of CMB for subsequent cerebral hemorrhage. 


\section{Materials and Methods}

\section{Data Source}

The moyamoya database was established at the University of Texas Southwestern Medical Center by including patients with an ICD9 code of MMD (437.5) or MMS (437.5) or cerebrovascular occlusive disease (437.9) retrospectively from 2007 to 2015. After IRB approval, screening for patients with MMD/MMS was performed daily among patients admitted to the Zale Lipshy University Hospital for prospective inclusion into the database.

\section{Study Population}

All patients with a confirmed diagnosis of MMD and MMS were identified and included in this study. A total of 156 patients were identified, out of which 76 were excluded due to unavailability or poor quality of GRE/SWI images. An additional 13 patients were also excluded due to concurrent atherosclerotic disease, leaving 67 total patients for analysis. The MR images were independently reviewed by 2 neuroradiologists and 1 vascular neurologist. CMB were defined according to previous criteria [19]. The patients were divided into 2 groups based on the presence/absence of $\mathrm{CMB}(\mathrm{CMB}+/ \mathrm{CMB}-)$. In $\mathrm{CMB}+$ patients, the number and location of $\mathrm{CMB}$ were identified. Patients were followed up by telephone interviews and by reviewing the electronic medical records of outpatient clinic visits, emergency room visits, and hospital admissions. Patient data collected included demographic data, stroke risk factors, history of bypass surgeries, and subsequent neurovascular events (TIA and ischemic and/or hemorrhagic strokes). The follow-up length was defined as the date between the presenting symptoms and the date of the final phone interview or the last date of electronic medical record system review.

\section{Statistical Analysis}

The patient variables analyzed included gender, race, age, disease type (bilateral MMD [MMD], unilateral MMD [uMMD], and MMS), age at diagnosis, presenting symptoms (ischemic events [ischemic stroke or TIA], hemorrhagic events [intracerebral hemorrhage or subarachnoid hemorrhage], or both, and others), comorbidities (hypertension, diabetes, hyperlipidemia, and thyroid diseases), smoking status, bypass (direct or indirect) surgery, follow-up strokes, and follow-up duration. Categorical variables were analyzed using a 2 -tailed $\chi^{2}$ test or Fisher's exact test. Continuous variables were analyzed using an independent $t$ test. OR, as a relative measure of risk, were calculated to indicate the increase in likelihood, for patients within a certain group, of developing CMB compared to those within the reference group. Univariate and multivariate logistic regressions were used to identify risk factors associated with developing $\mathrm{CMB}$ and whether CMB were associated with a risk of developing subsequent strokes. All tests were 2-sided, and $p<0.05$ was considered statistically significant. All analyses were performed using SAS 9.2 and IBM SPSS Statistics.

\section{Results}

\section{Characteristics of the Study Population}

A total of 67 patients were included in the final analysis, of whom $55(82 \%)$ were females with a mean age at the time of diagnosis of 36 years. GRE and SWI sequences were obtained in 59 and 8 patients, respectively. Full demographic details are listed in Table 1. We enrolled 45 patients (67\%) with MMD, 19 patients (28\%) with uMMD, and 3 patients (4\%) with MMS. The majority of the patients were Caucasian $(39,58 \%)$, while the rest were Asian $(8,12 \%)$, 
Khan et al.: Prevalence of and Risk Factors for CMB in MMD

Table 1. Characteristics of and differences between patients with/without CMB

\begin{tabular}{|c|c|c|c|c|c|c|}
\hline & \multicolumn{4}{|l|}{$\mathrm{CMB}$} & \multirow[t]{3}{*}{ Total, $n(\%)$} & \multirow[t]{3}{*}{$p$ value } \\
\hline & \multicolumn{2}{|l|}{ no } & \multicolumn{2}{|l|}{ yes } & & \\
\hline & $n$ & $\%$ & $n$ & $\%$ & & \\
\hline Subjects & 56 & 84 & 11 & 16 & $67(100)$ & \\
\hline Female gender & 49 & 88 & 6 & 55 & $55(82)$ & $0.021^{\mathrm{a}}$ \\
\hline Mean age at diagnosis, years & 34.1 & & 44.0 & & & $0.0236^{\mathrm{c}}$ \\
\hline \multicolumn{7}{|l|}{ Race } \\
\hline Asian & 7 & 12.5 & 1 & 9.1 & $8(11.9)$ & $0.489^{\mathrm{b}}$ \\
\hline Black & 6 & 10.7 & 3 & 27.3 & $9(13.4)$ & \\
\hline Caucasian & 33 & 58.9 & 6 & 54.5 & $39(58.2)$ & \\
\hline Hispanic & 10 & 17.9 & 1 & 9.1 & $11(16.4)$ & \\
\hline \multicolumn{7}{|l|}{ Moyamoya type } \\
\hline MMD or UMMD & 52 & 92.9 & 11 & 100 & $63(94.0)$ & $0.479^{\mathrm{a}}$ \\
\hline MMS & 4 & 7.1 & 0 & 0.0 & $4(6.0)$ & \\
\hline \multicolumn{7}{|c|}{ Presentation on diagnosis (symptomatic) } \\
\hline Ischemic & 45 & 80.4 & 4 & 36.4 & $49(73.1)$ & $0.006^{\mathrm{a}}$ \\
\hline Hemorrhagic & 5 & 8.9 & 4 & 36.4 & $9(13.4)$ & $0.034^{\mathrm{a}}$ \\
\hline Other & 6 & 10.7 & 3 & 27.3 & $9(13.4)$ & \\
\hline \multicolumn{7}{|l|}{ Comorbidity } \\
\hline Hypertension & 27 & 48.2 & 6 & 54.5 & $33(49.3)$ & $0.478^{\mathrm{a}}$ \\
\hline Diabetes mellitus & 10 & 17.9 & 2 & 18.2 & $12(17.9)$ & $0.634^{\mathrm{a}}$ \\
\hline Hyperlipidemia & 20 & 35.7 & 5 & 45.5 & $25(37.3)$ & $0.387^{\mathrm{a}}$ \\
\hline Thyroid disease & 9 & 16.1 & 1 & 9.1 & $10(14.9)$ & $0.480^{\mathrm{a}}$ \\
\hline Smoking & 15 & 26.8 & 8 & 72.7 & $23(34.3)$ & $0.006^{\mathrm{a}}$ \\
\hline Bypass surgery & 30 & 53.6 & 7 & 63.6 & 37 (55.2) & $0.392^{\mathrm{a}}$ \\
\hline
\end{tabular}

${ }^{\mathrm{a}}$ Fisher's exact test. ${ }^{\mathrm{b}} \chi^{2}$ test. ${ }^{\mathrm{c}}$ Student's $t$ test.

Black $(9,13 \%)$, or Hispanic $(11,16 \%)$. At the time of diagnosis, the majority of the patients presented with acute ischemic stroke or TIA (49; 73\%), while 9 patients (13\%) presented with hemorrhagic stroke. The remainder had other presentations such as seizures or headache. Of the 67 patients, 37 (55\%) underwent bypass surgery either during the initial hospital stay or during the following-up period.

\section{Predictors of Development of CMB}

Of the 67 enrolled patients, $11(16 \%)$ were found to have CMB. Males had a statistically significant higher OR of 1.76 (95\% CI 1.40-24.3, $p=0.021$ ) for having CMB compared to females. Other significant factors identified included age of diagnosis (mean age of CMB+ vs. CMB- patients: 44 vs. 34 years, respectively, $\mathrm{p}=0.0236)$, and smoking $(p=0.006)$. Presentation of ischemic events was associated with a lower risk of CMB (OR 1.97; 95\% CI 1.78-28.9, $p=0.006$ ) and, independently, $\mathrm{CMB}+$ was identified as significantly increased in patients presenting with hemorrhagic events $(p=0.034)$. Factors not found to be clinically significant included race, etiology of MMD, comorbid medical conditions (hypertension, diabetes, hyperlipidemia, and thyroid disease), and bypass surgery. Logistic regression with multivariate analysis (gender, age at diagnosis, smoking, and hemorrhagic stroke) found that gender and age at diagnosis remained statistically significant variables ( $p=0.016$ and 0.023 , respectively). 


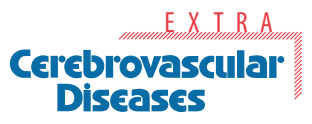

Table 2. Outcome measures

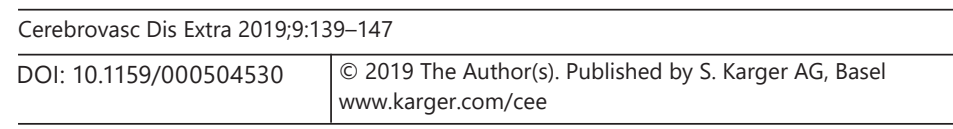

Khan et al.: Prevalence of and Risk Factors for CMB in MMD

\begin{tabular}{|c|c|c|c|c|}
\hline & \multicolumn{2}{|l|}{ CMB } & \multirow[t]{3}{*}{ Total } & \multirow[t]{3}{*}{$p$ value } \\
\hline & no & yes & & \\
\hline & $n, \%$ & $n, \%$ & & \\
\hline \multicolumn{5}{|l|}{ Follow-up stroke } \\
\hline None & $\begin{array}{l}40 \\
(72.2)\end{array}$ & $\begin{array}{c}8 \\
(80.0)\end{array}$ & $48(73.8)$ & $0.789^{\mathrm{a}}$ \\
\hline Ischemic & $\begin{array}{l}13 \\
(23.6)\end{array}$ & $\begin{array}{c}2 \\
(20)\end{array}$ & $15(23.1)$ & \\
\hline Hemorrhagic & $\begin{array}{c}2 \\
(3.6)\end{array}$ & $\begin{array}{c}0 \\
(0.0)\end{array}$ & $2(3.1)$ & \\
\hline \multicolumn{5}{|l|}{ Follow-up } \\
\hline Patients, $n$ & 55 & 9 & 64 & \\
\hline Mean length, months & 46.7 & 45.5 & & $0.939^{\mathrm{b}}$ \\
\hline
\end{tabular}

Values are presented as numbers (\%) unless otherwise stated. ${ }^{\text {a }}$ Fisher's exact test. ${ }^{\mathrm{b}}$ Student's $t$ test.

Follow-Up of Cohorts

Table 2 summarizes the outcome measures. During follow-up of the $10 \mathrm{CMB}+$ patients $(1$ patient was not followed up), 8 (80\%) did not have new strokes, while new ischemic events occurred in $2(20 \%)$ patients. None had new cerebral hemorrhagic events. Out of the 55 CMBpatients (1 patient was not followed up), 40 (72.7\%) had no new strokes, while $13(23.6 \%)$ had new ischemic events and $2(3.6 \%)$ had new cerebral hemorrhages. Analysis revealed that $\mathrm{CMB}+$ was not associated with subsequent ischemic or hemorrhagic events $(p=0.79)$. Table 3 summarizes the clinical profile of each $\mathrm{CMB}+$ patient.

\section{Discussion/Conclusion}

To our knowledge, this is the first report of the prevalence, risk factors, and predictive value of CMB in the development of subsequent stroke in a predominantly non-Asian MMD/ MMS patient population in the USA. The significance of CMB has been studied previously in Asian patients with MMD and MMS [20-22]. The first report identified asymptomatic CMB in $14.8 \%$ of patients. Subsequent studies reported the presence of CMB in up to $44 \%$ of patients with ischemic MMD and 43\% of patients with hemorrhagic MMD [23-25]. Conversely, in patients with MMD, CMB have also been identified as a significant risk factor for developing subsequent hemorrhagic stroke [26-28]. A recent meta-analysis of 245 MMD patients from 4 Japanese studies reported a CMB rate of 14.8-51.9\% [28]. In our study, 11 out of 67 (16\%) patients were found to have CMB. A similar frequency (12.9\%) was reported in a study in a European moyamoya population [18]. These values are on the lower end of the range cited in the meta-analysis of Asian populations [28]. Of these 11 patients, a total of $20 \mathrm{CMB}$ were identified, with most patients having only 1-2 CMB each.

\section{Risk Factors for the Development of CMB}

Ishikawa et al. [23] found no correlation of CMB with patient age, duration from disease onset, or disease diagnosis. Furthermore, Sun et al. [27] reported that gender did not correlate with CMB. In our study, the average age was similar to values in previous studies. Our femaleto-male ratio was 4.6, which is also within the range of previous studies (i.e., 2.5-5.2). We saw a statistically significant difference, with male gender being a potential risk factor for devel- 


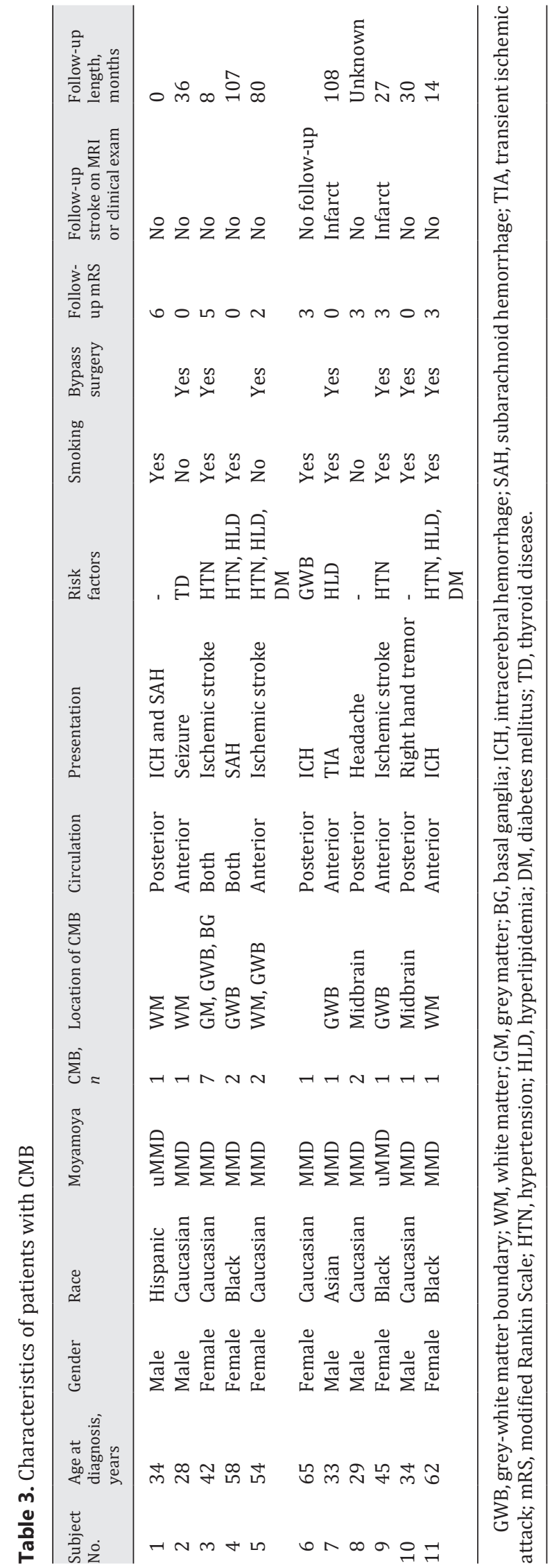


oping $\mathrm{CMB}(\mathrm{OR}=1.76, p=0.021)$. We also note that there was a statistically significant difference in the mean age at diagnosis between $\mathrm{CMB}+$ and $\mathrm{CMB}-$ patients; the age at diagnosis of the $\mathrm{CMB}+$ patients was significantly older than that of the CMB- patients by about 10 years ( 44 vs. 34 years, $p=0.024$ ). When multivariate analysis was done with logistic regression, both of these variables remained statistically significant. However, these results should be interpreted cautiously. The sample size is relatively small for multivariate analysis. Based on our data, it is not possible to determine whether this is simply clinical progression of the moyamoya vasculopathy giving the patient more time to develop CMB or whether older patients themselves are at risk of CMB due to other etiologies. We did not specifically see the higher percentage of Caucasian race as being clinically or statistically significant as a risk factor for the development of CMB; however, we note that this study was not powered to evaluate that question.

In our cohort, we did not identify other factors as additional independent predictors of the development of CMB. These factors include smoking and hemorrhagic events at presentation. Sun et al. [27] reported that hemorrhagic onset, diabetes, and blood pressure did not correlate with CMB. In a study by Ding et al. [29], smoking was found to be significantly associated with an increased risk of lobar CMB exclusively but not for deep CMB. In our cohort, we noted that comorbidities such as hypertension, diabetes mellitus, hyperlipidemia, and thyroid disease, all of which are classically associated with an increased risk of neurovascular events, were not found to be significant in the development of CMB.

\section{Strokes in $M M D / M M S$ with and without $C M B$}

In our cohort, the majority (73\%) of patients presented with ischemic events at the time of diagnosis. We detected a statistically significant difference in $\mathrm{CMB}+$ patients having having ischemic events on presentation compared to CMB-. Furthermore, we noted an increase in hemorrhagic events at onset in our $\mathrm{CMB}+$ patients, but it was not found to be statistically significant on multivariate analysis. This data seems to be in contrast with a recent metaanalysis that reported that the incidence of CMB was higher in hemorrhagic onset MMD in an Asian population [28].

In a prospective cohort study, Kuroda et al. [26] concluded that the presence of silent CMB was a significant predictor for subsequent hemorrhagic stroke in an Asian moyamoya population. However, we found no association of subsequent ischemic or hemorrhagic events with the presence of CMB during follow-up in this predominantly non-Asian population. Sun et al. [27] presented another prospective study of a Chinese population where 85 adult patients with MMD were followed, with CMB in $28.2 \%$ of the patients. Similar to our study, they found no statistically significant association between CMB and subsequent infarction/ hemorrhage. However, in their study, patients with CMB foci in the deep and periventricular white matter had a statistically higher likelihood of subsequent intraventricular hemorrhage compared to patients without $\mathrm{CMB}$ foci and with $\mathrm{CMB}$ in other regions [27]. In our CMB cohort, we noted that none of our patients had hemorrhagic events at follow up; rather 2 out of 10 patients ( 1 was not followed up) had subsequent ischemic strokes, but this was not statistically significant compared to our cohort without CMB. In these 2 patients, CMB foci were located in the grey-white matter boundary regions.

\section{Critical Evaluation of This Study}

The power of our study is limited by the small patient population. The moyamoya database did not contain information of certain medications such as nonsteroidal anti-inflammatory drugs, serotonin-norepinephrine reuptake inhibitors, and serotonin-specific reuptake inhibitors, which may affect the risk of bleeding and potentially affect the results. There was some heterogeneity in imaging data, with some patients having standard GRE sequences and 
others having SWI, which is known to be significantly more sensitive to the detection of CMB [30]. Lastly, our study is retrospective and the follow-up period might be too short to accurately determine the association between $\mathrm{CMB}$ and subsequent strokes.

In conclusion, the prevalence of CMB in MMD/MMS patients in the USA is lower than in Asian populations. The risk factors that were identified to be associated with $\mathrm{CMB}+$ were male gender and an older age at diagnosis. CMB+ was not associated with an increased risk of subsequent cerebral hemorrhage or ischemic stroke in this non-Asian population. Further studies with a larger number of patients and a longer follow-up period are needed to confirm these findings.

\section{Statement of Ethics}

IRB approval was obtained for database establishment. Since the data was analyzed retrospectively from the database and in a de-identified fashion, individual patient consent was not required.

\section{Disclosure Statement}

The authors have no conflicts of interest to declare.

\section{Funding Sources}

The research reported in this publication was supported by the National Center for Advancing Translational Sciences of the National Institutes of Health under award No. UL1TR001105. The content is solely the responsibility of the authors and does not necessarily represent the official views of the National Institutes of Health. Study data were collected and managed using REDCap electronic data capture tools hosted at the University of Texas Southwestern Medical Center.

\section{Author Contributions}

Nadeem I. Khan: data analysis and interpretation and drafting of this paper. Ali A. Saherwala: statistical analysis, interpretation of data, and writing of this paper. Mo Chen: data collection, analysis, and writing of this paper. Sepand Salehian: data collection, image interpretation, and critical review of this paper. Hisham Salahuddin: data collection and writing of this paper. Babu G. Welch: data analysis and writing of this paper. Marco C. Pinho: image interpretation, data collection and analysis, and review of this paper. Ty Shang was involved in conception and design of this study, data collection and analysis, and writing of this paper. The final version of this paper was reviewed and approved by all of the authors.

\section{References}

1 Uchino K, Johnston SC, Becker KJ, Tirschwell DL. Moyamoya disease in Washington State and California. Neurology. 2005 Sep;65(6):956-8.

2 Miyawaki S, Imai H, Takayanagi S, Mukasa A, Nakatomi H, Saito N. Identification of a genetic variant common to moyamoya disease and intracranial major artery stenosis/occlusion. Stroke. 2012 Dec;43(12):3371-4.

3 Lee SJ, Ahn JY. Stenosis of the proximal external carotid artery in an adult with moyamoya disease: moyamoya or atherosclerotic change? Neurol Med Chir (Tokyo). 2007 Aug;47(8):356-9. 
Khan et al.: Prevalence of and Risk Factors for CMB in MMD

4 Sharfstein SR, Ahmed S, Islam MQ, Najjar MI, Ratushny V. Case of moyamoya disease in a patient with advanced acquired immunodeficiency syndrome. J Stroke Cerebrovasc Dis. 2007 Nov-Dec;16(6):268-72.

5 Fryer RH, Anderson RC, Chiriboga CA, Feldstein NA. Sickle cell anemia with moyamoya disease: outcomes after EDAS procedure. Pediatr Neurol. 2003 Aug;29(2):124-30.

6 Dobson SR, Holden KR, Nietert PJ, Cure JK, Laver JH, Disco D, et al. Moyamoya syndrome in childhood sickle cell disease: a predictive factor for recurrent cerebrovascular events. Blood. 2002 May;99(9):3144-50.

7 Kirkham FJ, DeBaun MR. Stroke in children with sickle cell disease. Curr Treat Options Neurol. 2004 Sep;6(5): 357-75.

8 Rosser TL, Vezina G, Packer RJ. Cerebrovascular abnormalities in a population of children with neurofibromatosis type 1. Neurology. 2005 Feb;64(3):553-5.

9 Imaizumi M, Nukada T, Yoneda S, Takano T, Hasegawa K, Abe H. Tuberous sclerosis with moyamoya disease. Case report. Med J Osaka Univ. 1978 Mar;28(3-4):345-53.

10 Garcia JC, Roach ES, McLean WT. Recurrent thrombotic deterioration in the Sturge-Weber syndrome. Childs Brain. 1981;8(6):427-33.

11 de Borchgrave V, Saussu F, Depre A, de Barsy T. Moyamoya disease and Down syndrome: case report and review of the literature. Acta Neurol Belg. 2002 Jun;102(2):63-6.

12 Manjila S, Miller BR, Rao-Frisch A, Otvos B, Mitchell A, Bambakidis NC, et al. Moyamoya disease associated with asymptomatic mosaic Turner syndrome: a rare cause of hemorrhagic stroke. J Stroke Cerebrovasc Dis. 2014 May-Jun;23(5):1242-4.

13 Carlson CB, Harvey FH, Loop J. Progressive alternating hemiplegia in early childhood and basal arterial stenosis and telangiectasia (moyamoya syndrome). Neurology. 1973 Jul;23(7):734-44.

14 Kikuta K, Takagi Y, Nozaki K, Okada T, Hashimoto N. Histological analysis of microbleed after surgical resection in a patient with moyamoya disease. Neurol Med Chir (Tokyo). 2007 Dec;47(12):564-7.

15 Hallemeier CL, Rich KM, Grubb RL Jr, Chicoine MR, Moran CJ, Cross DT 3rd, et al. Clinical features and outcome in North American adults with moyamoya phenomenon. Stroke. 2006 Jun;37(6):1490-6.

16 Gross BA, Du R. The natural history of moyamoya in a North American adult cohort. J Clin Neurosci. 2013 Jan; 20(1):44-8.

17 Kim BJ, Lee SH. Prognostic impact of cerebral small vessel disease on stroke outcome. J Stroke. 2015 May; 17(2):101-10

18 Wenz H, Wenz R, Maros M, Ehrlich G, Al-Zghloul M, Groden C, et al. Incidence, locations, and longitudinal course of cerebral microbleeds in european moyamoya. Stroke. 2017 Feb;48(2):307-13.

19 Greenberg SM, Vernooij MW, Cordonnier C, Viswanathan A, Al-Shahi Salman R, Warach S, et al.; Microbleed Study Group. Cerebral microbleeds: a guide to detection and interpretation. Lancet Neurol. 2009 Feb;8(2): 165-74.

20 Wakai K, Tamakoshi A, Ikezaki K, Fukui M, Kawamura T, Aoki R, et al. Epidemiological features of moyamoya disease in Japan: findings from a nationwide survey. Clin Neurol Neurosurg. 1997 Oct;99 Suppl 2:S1-5.

21 Baba T, Houkin K, Kuroda S. Novel epidemiological features of moyamoya disease. J Neurol Neurosurg Psychiatry. 2008 Aug;79(8):900-4.

22 Amlie-Lefond C, Zaidat O0, Lew SM. Moyamoya disease in early infancy: case report and literature review. Pediatr Neurol. 2011 Apr;44(4):299-302.

23 Ishikawa T, Kuroda S, Nakayama N, Terae S, Kudou K, Iwasaki Y. Prevalence of asymptomatic microbleeds in patients with moyamoya disease. Neurol Med Chir (Tokyo). 2005 Oct;45(10):495-500.

24 Kikuta K, Takagi Y, Nozaki K, Hanakawa T, Okada T, Mikuni N, et al. Asymptomatic microbleeds in moyamoya disease: T2*-weighted gradient-echo magnetic resonance imaging study. J Neurosurg. 2005 Mar;102(3): 470-5.

25 Mori N, Miki Y, Kikuta K, Fushimi Y, Okada T, Urayama S, et al. Microbleeds in moyamoya disease: susceptibility-weighted imaging versus T2*-weighted imaging at 3 Tesla. Invest Radiol. 2008 Aug;43(8):574-9.

26 Kuroda S, Kashiwazaki D, Ishikawa T, Nakayama N, Houkin K. Incidence, locations, and longitudinal course of silent microbleeds in moyamoya disease: a prospective T2*-weighted MRI study. Stroke. 2013 Feb;44(2): 516-8.

27 Sun W, Yuan C, Liu W, Li Y, Huang Z, Zhu W, et al. Asymptomatic cerebral microbleeds in adult patients with moyamoya disease: a prospective cohort study with 2 years of follow-up. Cerebrovasc Dis. 2013;35(5):46975.

28 Qin Y, Ogawa T, Fujii S, Shinohara Y, Kitao S, Miyoshi F, et al. High incidence of asymptomatic cerebral microbleeds in patients with hemorrhagic onset-type moyamoya disease: a phase-sensitive MRI study and metaanalysis. Acta Radiol. 2015 Mar;56(3):329-38.

29 Ding J, Sigurdsson S, Garcia M, Phillips CL, Eiriksdottir G, Gudnason V, et al. Risk Factors Associated With Incident Cerebral Microbleeds According to Location in Older People: The Age, Gene/Environment Susceptibility (AGES)-Reykjavik Study. JAMA Neurol. 2015 Jun;72(6):682-8.

30 Cheng AL, Batool S, McCreary CR, Lauzon ML, Frayne R, Goyal M, et al. Susceptibility-weighted imaging is more reliable than T2*-weighted gradient-recalled echo MRI for detecting microbleeds. Stroke. 2013 Oct; 44(10): 2782-6. 